\title{
A 10-year prospective surveillance of Mycobacterium tuberculosis drug resistance in France 1995-2004
}

\author{
P.M. Khuê,*\#, C. Truffot-Pernot*,\#, J. Texier-Maugein ${ }^{\star}$, V. Jarlier*,\# and \\ J. Robert*, ${ }^{*}$ on behalf of the AZAY-Mycobacteria Study Group
}

ABSTRACT: Drug resistance surveillance and trend monitoring resistance rates bring some insights into tuberculosis (TB) control. The current study reports the characteristics of TB and drug resistance during a 10-yr prospective surveillance of culture-positive TB in France.

Data for the current study was collected from 1995-2004 via a sentinel network of laboratories from university hospitals that complied with the international recommendations for the surveillance of drug resistance. Susceptibility test results were performed in each individual laboratory.

Data on 13,283 patients were collected during the 10-yr period, $49 \%$ of whom had been born in France, $10 \%$ were HIV co-infected and $8 \%$ had previously been treated. As expected, previously treated and HIV co-infected patients were more likely to harbour resistant strains, especially rifampicin (RMP)-resistant strains. Among new patients, the mean resistance rate to at least one drug was $\mathbf{8 . 8 \%}$, and there was an upward trend in resistance to isoniazid and RMP (0.8-1\%) related to the increase in the proportion of patients who had been born outside of France (38$53 \%)$. Among previously treated patients, the mean resistance rate to one drug was $20.6 \%$ and there was no significant time trend in resistance rates.

The sentinel network provided valuable data on trends regarding the characteristics of tuberculosis and on drug resistance rates and reinforced the interest of analysing data by country of birth and history of treatment.

KEYWORDS: Isoniazid, resistance, rifampicin, surveillance, trend, tuberculosis

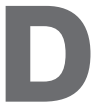
rug resistance surveillance is considered to be of major interest in tuberculosis (TB) surveillance as resistance may be a major threat to TB control. The prevalence of anti-TB drug resistance and especially multidrug resistance (MDR), i.e. resistance to at least isoniazid (INH) and rifampicin (RMP), is related to the proportion of previously treated cases [1]. Low resistance rates are associated with good management practices, such as short-course chemotherapy and directly observed treatments. For these reasons, the World Health Organization (WHO) and the International Union Against TB and Lung Disease issued guidelines for the surveillance of anti-TB drug resistance [2]. These guidelines were further modified, adapting to the epidemiological and technical situations found in European countries [3]. The main principles underlined in the guidelines are: 1) to report drug resistance on a representative number of cases; 2 )

For editorial comments see page 825 . to stratify results on patients' previous treatment histories; and 3) to stratify results on patients' origin. The main objectives of this surveillance were to monitor the trends for TB infection and identify high-risk populations.

In France during 2004, approximately 5,500 cases of TB were reported to the French Health Authorities through the national mandatory notification system and the incidence of TB in metropolitan France was given as 9.2 per 100,000 inhabitants [4]. Consequently, France is considered to be a low incidence country for TB. There is currently no national directly observed therapy programme, although the rate for successful TB treatment is estimated at $>85 \%$. TB notification is mandatory throughout France, although until recently no data on drug resistance had been collected through the mandatory system. The surveillance of drug resistance, established in the early 1960s at the Pasteur Institute (Paris, France) [5], stopped in the early
AFFILIATIONS

*Laboratory of Bacteriology and Hygiene, Pierre et Marie Curie-Paris6 University and,

\#National Reference Centre for Mycobacteria and Antituberculosis Drug Resistance, APHP PitiéSalpêtrière Hospital, Paris, and "Laboratory of Bacteriology, Haut Lévêque Hospital, Bordeaux, France.

CORRESPONDENCE

J. Robert

Laboratoire de Bactériologie-Hygiène UFR de Médecine Pierre et Marie Curie Paris 6 91 Boulevard de l'hôpital 75634 Paris Cedex 13 France

Fax: 33145827577

E-mail: jrobert@chups.jussieu.fr

Received:

March 282007

Accepted after revision:

June 072007

SUPPORT STATEMENT

P.M. Khuê was supported by a grant from Agence Universitaire de la Francophonie and a grant from La Société de Pathologie Exotique de Langue Française.

STATEMENT OF INTEREST None declared.

European Respiratory Journal Print ISSN 0903-1936 Online ISSN 1399-3003 
1970s. Surveillance resumed in 1995, thanks to a new laboratory-based network of university hospitals [6]. Data collected through this network are not linked to the mandatory notification system and therefore the network is considered as a sentinel surveillance system. In a previous report [6], the current authors described the results of the first 3 yrs of the surveillance system. The present authors found patients' characteristics were similar to those of patients notified through the mandatory notification system and that drug resistance rates for France ranked it in the lower range of European countries, when considering resistance rates.

The current study presents the characteristics for TB and drugresistance rates during a 10-yr prospective surveillance of culture-positive TB. The surveillance was performed by a network of university hospitals complying with international recommendations for the surveillance of drug resistance. The present authors' analyses focused on drug resistance in patients born outside of France, HIV co-infected patients and time trends over the $10-y r$ period.

\section{MATERIALS AND METHODS}

The network of microbiologists from the university hospitals, called the AZAY-Mycobacteria Study Group, was established in 1994 and surveillance began in 1995. After 3 yrs of consolidation [6], the 19 initial laboratories were progressively joined by new laboratories in order to increase geographical coverage. At the end of the study period in 2004, the network included 33 laboratories.

All culture-positive TB patients diagnosed in each laboratory from the network, during the 10-yr period, were included in the surveillance. Microbiologists prospectively collected the following data: basic demographical data; place of birth; site of $\mathrm{TB}$, by clinical examination, chest radiographs and laboratory results; HIV co-infection; prior history of treatment with antiTB drugs and microbiological results. Susceptibility to first line antimicrobials, i.e. INH, RMP, ethambutol (EMB) and streptomycin (STM), was conducted independently by each laboratory; except for a few laboratories with too few numbers of cases that transmitted positive-culture slants for susceptibility testing to another laboratory within the network. The method used for susceptibility testing relied on the proportion method, either on Löwenstein-Jensen (LJ) [7] or liquid media [8]. Criteria for drug resistance on LJ medium were $\geqslant 1 \%$ colony growth at 28 and 40 days compared with the drug-free control medium at the following drug concentrations: 0.2, 40, 2 and $4 \mathrm{mg} \cdot \mathrm{L}^{-1}$ for INH, RMP, EMB and STM, respectively [7]. For the BACTEC MGIT 960 system (Becton-Dickinson, Baltimore, MD, USA), critical drug concentrations were 0.1 and $0.4 \mathrm{mg} \cdot \mathrm{L}^{-1}$ for INH, 1 and $4 \mathrm{mg} \cdot \mathrm{L}^{-1}$ for STM, and 1 and $5 \mathrm{mg} \cdot \mathrm{L}^{-1}$ for RMP and $\mathrm{EMB}$, respectively. Data on pyrazinamide were not collected because of the uncertainties found in its interpretation [8]. Quality of susceptibility results was assessed by internal and external quality controls. The National Reference Centre (NRC) participated in the proficiency testing of the susceptibility of Mycobacterium tuberculosis organised by WHO. The NRC organised blind quality controls in the network. This was undertaken by sending strains of the bacterium, with a well-defined resistant pattern, to each participating laboratory. Results of these blind controls were satisfactory with regards to the WHO standards.
Patients were classified as new, previously treated or chronic cases according to international definitions [7]. Patients with unknown HIV status were grouped with HIV-negative patients for analysis. MDR was defined by resistance to a minimum of INH and RMP.

Data were collected on standardised forms, computerised and analysed using computer software. Categorical variables were compared by using Chi-squared tests or Fisher's exact test. Trends were analysed by using the Chi-squared tests for trend. To determine the independent importance of major risk factors for drug resistance, the current authors developed unconditional logistic regression models with bootstrapping. Age was dichotomised at the median to be introduced in the models. Birthplace outside of France or the different French regions of birth were used alternatively for model building. All variables were included in the models and backward analysis was performed. Only the most parsimonious models, i.e. those having the most significance with the least variables, are presented. Model fitness was assessed by using the HosmerLemeshow test. The p-values are two-tailed unpaired and $\mathrm{p}<0.05$ was considered statistically significant.

\section{RESULTS}

\section{Population}

During the 10-yr surveillance period, the number of laboratories participating in the network increased from 17 in 1995 to 19 in 1998 and reached 33 in 2004. Consequently, the number of French metropolitan regions covered by the network increased from nine out of the 22 administrative regions in 1995 to 19 out of 22 administrative regions in 2004. The annual number of patients increased from 1,052 in 1995, remaining stable at $\sim 1,150$ patients until 2000, reaching 1,732 in 2004 . During the initial 3 yrs of the surveillance, the number of patients included in the surveillance represented a mean proportion of $10 \%$ from the total number of patients recorded each year by the French national mandatory notification system for TB [4]. This proportion reached $29 \%$ during the latter 3 yrs of the surveillance [4]. The Paris area included $43 \%$ of the patients over the $10-y r$ period.

A total of 13,283 patients were included during the $10-y r$ surveillance, of whom $8,642(65 \%)$ were male (table 1$)$. The mean age was 48.6 yrs and $72 \%$ of the patients were aged between 15-64 yrs. Half of the patients $(6,554$ out of 13,283 ; $49 \%$ ) were born in France, whilst 5,806 (44\%) were born outside of France, with the remaining 923 (7\%) patients having an unknown country of birth. The proportion of HIV coinfected patients was $10 \%$. Patients born outside of France were more likely to be HIV co-infected $(13.6 \%)$ than patients who had been born in France $(7.5 \%, p<0.01)$. A total of 10,707 $(80 \%)$ patients were new or previously untreated cases, 1,019 $(8 \%)$ had previously received anti-TB drugs for $>1$ month, and treatment history was unknown for the remaining 1,557 (12\%) patients. The majority of patients $(70 \%)$ had pulmonary TB, including 53\% smear-positive cases, $22 \%$ had extra-pulmonary $\mathrm{TB}$ and the remaining $8 \%$ of patients had combined pulmonary and extra-pulmonary TB. HIV-positive patients with extrapulmonary TB were more likely to be smear-positive than HIVnegative patients (44 versus $23 \% ; \mathrm{p}<0.001$ ). In contrast, the difference in positive smears for pulmonary $\mathrm{TB}$ was not 
significant between HIV-positive and HIV-negative patients (57 versus $53 \%$; $\mathrm{p}=0.06$ ).

During the 10-yr surveillance period there was a significant increase in the proportion of patients born outside of France, from $38 \%$ in 1995 to $53 \%$ in 2004 ( $p<0.001$, Chi-squared test for trend; fig. 1). Overall, the most frequent regions of birth for the patients born outside of France were sub-Saharan Africa (16.6\%), Maghreb (12.5\%) and Asia (6.9\%). During the 10-yr study period, there was a constant increase in the proportion of patients who originated from sub-Saharan Africa (12.0-21.9\%; $\mathrm{p}<0.001)$. In addition, a significant increase was observed in the first part of the study period for patients born in Maghreb $(9.1 \%$ in 1995 to $14.0 \%$ in $1998 ; \mathrm{p}<0.001)$ and in Asia $(4.8 \%$ in 1995 to $7.8 \%$ in $2000 ; \mathrm{p}<0.001)$, but these proportions remained

\section{TABLE 1 Characteristics of the study population}

\begin{tabular}{|c|c|}
\hline \multicolumn{2}{|l|}{ Treatment history } \\
\hline Previously untreated & $10707(80)$ \\
\hline Previously treated & $1019(8)$ \\
\hline Unknown & $1557(12)$ \\
\hline \multicolumn{2}{|l|}{ Country of birth } \\
\hline France & $6554(49)$ \\
\hline Other & $5806(44)$ \\
\hline Western Europe & 422 (3.2) \\
\hline Eastern Europe & $216(1.6)$ \\
\hline Maghreb & $1654(12.5)$ \\
\hline Sub-Saharan Africa & 2207 (16.6) \\
\hline Asia & $921(6.9)$ \\
\hline All other countries & $193(1.5)$ \\
\hline Unknown & $923(7)$ \\
\hline HIV+ status & $1326(10)$ \\
\hline \multicolumn{2}{|l|}{ Age yrs } \\
\hline $0-14$ & $176(1)$ \\
\hline $15-64$ & $9530(72)$ \\
\hline$\geqslant 65$ & $3473(27)$ \\
\hline \multicolumn{2}{|l|}{ Tuberculosis $\#$} \\
\hline Pulmonary" & $9262(70)$ \\
\hline $\mathrm{SM}+, \mathrm{HIV}+$ & $457(5)$ \\
\hline $\mathrm{SM}+, \mathrm{HIV}-$ & $4445(48)$ \\
\hline SM-, HIV+ & $350(4)$ \\
\hline SM-, HIV- & 3929 (43) \\
\hline Extra-pulmonary $^{+}$ & $2870(22)$ \\
\hline $\mathrm{SM}+, \mathrm{HIV}+$ & $91(3)$ \\
\hline SM+, HIV- & $591(21)$ \\
\hline SM-, HIV+ & $117(4)$ \\
\hline SM-, HIV- & $1992(72)$ \\
\hline Combined & $1125(8)$ \\
\hline \multicolumn{2}{|c|}{ Diagnosis for each region of France } \\
\hline Paris area & $5732(43)$ \\
\hline North west & $2208(17)$ \\
\hline North east & $1074(8)$ \\
\hline South west & $2541(19)$ \\
\hline South east & $1726(13)$ \\
\hline Total & $13283(100)$ \\
\hline
\end{tabular}

Data are presented as $n(\%) .+$ : positive; -: negative. \#: Tuberculosis site was unknown for 26 patients; ": smear (SM) result was unknown for 81 patients; +. SM result was unknown for 79 patients. stable afterwards for both populations $(p=0.42$ and $p=0.32$ respectively). The proportion of patients with a previous history of treatment decreased from 11 to $8 \%(\mathrm{p}<0.001)$. The proportion of HIV co-infected patients remained stable at $\sim 10 \%$ during the $10-y r$ period $(p=0.62)$. There was no difference in trends observed for all the above characteristics between laboratories participating in the surveillance since the beginning of the programme $(n=19$, referred to as the initial laboratories) and the laboratories that joined the network afterwards ( $n=14$, referred to as the new laboratories).

\section{Primary resistance}

The overall resistance rate to at least one first line drug or STM in new cases was $8.8 \%$. STM resistance was the most frequent $(6.6 \%)$, followed by INH resistance (4.2\%). MDR was observed in $0.7 \%(n=78)$ of the new cases (table 2$)$. Resistance to at least one drug was more likely in patients born outside of France $(11 \%)$ than in French-born patients $(7 \% ; p=0.001)$. Among those patients who had been born in France, resistance to at least RMP or EMB was more frequent in HIV co-infected patients ( 2.0 and $1.2 \%$, respectively) than in other patients $(0.4$ and $0.3 \%$, respectively; $p<0.01$ ). In addition, the resistance rate to RMP alone (monoresistance) was higher among HIV coinfected patients than among other patients (1.0 versus $0.04 \%$; $\mathrm{p}<0.001)$. There was no statistically significant difference among HIV co-infected patients and other patients for resistance to at least STM, INH or MDR. Compared with non-HIV co-infected patients, HIV co-infected patients born outside of France, had higher resistance rates to STM (10.6 versus $6.6 \%, \mathrm{p}<0.001)$, INH (9.2 versus $5.9 \%, \mathrm{p}=0.002)$, RMP ( 3.0 versus $1.1 \%, p<0.001)$ and MDR $(2.7$ versus $0.9 \% ; \mathrm{p}<0.001)$.

Factors independently associated with primary resistance to at least one of the four drugs in multivariate analysis (table 3 ) were: age $<45$ yrs (odds ratio (OR) 1.2); born outside of France (OR 1.5); and HIV co-infection (OR 1.4). Sub-Saharan Africa (OR 1.5) or Asia (OR 1.6) were the two regions of birth strongly associated with resistance, as compared with other regions when introduced in the logistic regression model in place of birth outside of France. Factors independently associated with

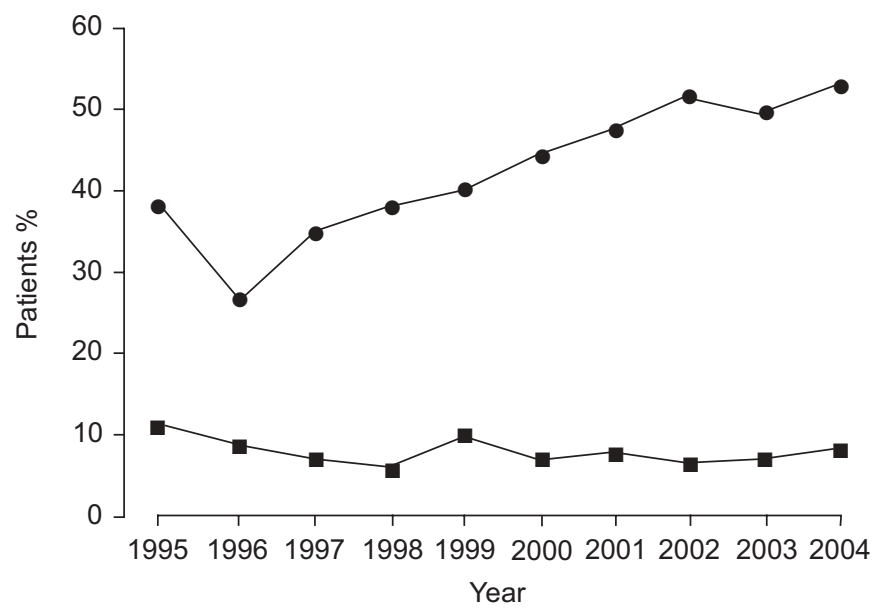

FIGURE 1. Evolution of the country of birth and previous history of treatment for tuberculosis for patients surveyed from 1995-2004 in France. • : patients born outside of France; $\mathbf{n}$ : previously treated patients 


\begin{tabular}{|c|c|c|c|c|c|}
\hline \multirow[t]{2}{*}{ Result from drug susceptibility testing } & \multirow[t]{2}{*}{ All patients } & \multicolumn{2}{|c|}{ Born in France } & \multicolumn{2}{|c|}{ Born outside of France } \\
\hline & & HIV- & HIV+ & HIV- & HIV+ \\
\hline Fully sensitive & 9769 (91.2) & $4731(93.4)$ & $368(90.9)$ & $3729(90.0)$ & $539(85.6)$ \\
\hline Any INH resistance & $452(4.2)$ & $108(2.1)$ & $14(3.5)$ & $245(5.9)$ & $58(9.2)$ \\
\hline Any RMP resistance & $92(0.9)$ & $18(0.4)$ & $8(2.0)$ & $44(1.1)$ & $19(3.0)$ \\
\hline Any EMB resistance & $61(0.6)$ & $16(0.3)$ & $5(1.2)$ & $30(0.7)$ & $7(1.1)$ \\
\hline Monoresistance & $674(6.3)$ & $278(5.5)$ & $29(7.2)$ & $273(6.6)$ & $47(7.5)$ \\
\hline STM & $450(4.2)$ & $217(4.3)$ & $16(4.0)$ & $157(3.8)$ & $28(4.4)$ \\
\hline INH+RMP resistance & $78(0.7)$ & $16(0.3)$ & $3(0.7)$ & $39(0.9)$ & $17(2.7)$ \\
\hline $\mathrm{INH}+\mathrm{RMP}$ & $24(0.2)$ & $3(0.1)$ & $1(0.2)$ & $15(0.4)$ & $5(0.8)$ \\
\hline $\mathrm{INH}+\mathrm{RMP}+\mathrm{EMB}$ & $8(0.1)$ & $1(<0.1)$ & $2(0.5)$ & $4(0.1)$ & $0(0.0)$ \\
\hline $\mathrm{INH}+\mathrm{RMP}+\mathrm{STM}$ & $33(0.3)$ & $8(0.2)$ & $0(0.0)$ & $15(0.4)$ & $9(1.4)$ \\
\hline $\mathrm{INH}+\mathrm{RMP}+\mathrm{EMB}+\mathrm{STM}$ & $13(0.1)$ & $4(0.1)$ & $0(0.0)$ & $5(0.1)$ & $3(0.5)$ \\
\hline INH+other resistance & $189(1.8)$ & $44(0.9)$ & $5(1.2)$ & $110(2.7)$ & $28(4.4)$ \\
\hline $\mathrm{INH}+\mathrm{STM}$ & $171(1.6)$ & $38(0.8)$ & $3(0.7)$ & $94(2.3)$ & $26(4.1)$ \\
\hline $\mathrm{INH}+\mathrm{EMB}$ & $10(0.1)$ & $4(0.1)$ & $1(0.2)$ & $12(0.3)$ & $1(0.2)$ \\
\hline INH+EMB+STM & $8(0.1)$ & $2(0.0)$ & $1(0.2)$ & $4(0.1)$ & $1(0.2)$ \\
\hline $\mathrm{RMP}+$ other resistance & $2(0.0)$ & $1(0.0)$ & $1(0.2)$ & 0 & 0 \\
\hline $\mathrm{RMP}+\mathrm{STM}$ & $2(0.0)$ & $1(0.0)$ & $1(0.2)$ & 0 & 0 \\
\hline
\end{tabular}

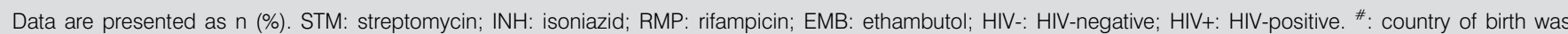
unknown for 462 patients.

MDR were place of birth outside France (OR 2.7) and HIV coinfection (OR 2.6). Younger age was not significantly associated with MDR (OR 1.6, p 0.11). Finally, when analysing risk factors for resistance to RMP alone, only HIV co-infection remained significantly associated with an increased risk (OR 8.9, 95\% confidence interval (CI) 2.9-27.6).
There was no significant time trend $(p=0.81)$ in the overall resistance to at least one drug, STM or RMP among new cases during the 10-yr surveillance (fig. 2). However, there was an upward trend in resistance to INH (3.7 to $4.3 \% ; \mathrm{p}=0.01)$, and in $\operatorname{MDR}(0.81$ to $1 \%, \mathrm{p}=0.02)$. When separately analysing the data from initial laboratories, an upward trend was observed for

TABLE 3 Multivariate analyses of risk factors for drug resistance to tuberculosis between 1995-2004 in France

\begin{tabular}{lcc} 
Characteristics & Resistance $\geqslant \mathbf{1}$ drug & Resistance to at least INH+RMP \\
\hline New patients & & $1.6(0.9-207)$ \\
Age $<45$ yrs & $1.2(1.01-1.4)$ & $2.7(1.5-5.0)$ \\
Born outside of France & $1.5(1.3-1.7)$ & $2.6(1.4-4.7)$ \\
HIV co-infection & $1.4(1.2-1.8)$ & \\
Previously treated patients & $1.9(1.3-2.8)$ & $2.0(1.1-3.6)$ \\
Age $<45$ yrs & $1.9(1.3-2.8)$ & $5.5(2.8-10.7)$ \\
Born outside of France & $1.9(1.2-2.9)$ & NS \\
HIV co-infection & $1.6(1.1-2.2)$ & NS \\
SM+ &
\end{tabular}

Data presented as odds ratio (95\% confidence interval). INH: isoniazid; RMP: rifampicin; SM+: smear positive; Ns: nonsignificant. 
MDR, but not for INH resistance. After stratification on the place of birth, no time trend was observed for INH and MDR, or for French-born or non-French-born patients.

\section{Secondary resistance}

The overall resistance to at least one first-line drug or STM in previously treated cases was $20.6 \%$. INH resistance was the most frequent $(14.0 \%)$, followed by STM resistance $(11.7 \%)$. Resistance to RMP was observed in $8.9 \%$ of the cases, and MDR resistance in $6.9 \%(n=70$; table 4$)$. Resistance to at least one drug was more likely in patients born outside of France $(29 \%)$ than in French-born patients $(14 \%, \mathrm{p}<0.001)$. Among French-born previously treated patients, resistance to at least RMP or to RMP alone was more frequent among HIV coinfected patients (28.6 and $22.4 \%$, respectively) than among other patients (2.2 and $0 \%$, respectively; $\mathrm{p}<0.001$ for both comparisons). There was no statistically significant difference among HIV co-infected patients and other patients for resistance to other drugs and MDR. As for French-born patients, HIV co-infected patients born outside of France had higher resistance rates to RMP alone $(8.6 \%)$ than non-HIV coinfected patients $(0 \% ; \mathrm{p}<0.001)$. No other statistical difference was observed among patients born outside of France after stratification on HIV status.

Factors independently associated with resistance to at least one of the four drugs among previously treated patients in multivariate analysis (table 3) were the same as those for new patients i.e. aged $<45$ yrs (OR 1.9), born outside of France (OR 1.9), and HIV co-infection (OR 1.9). In addition, smearpositive patients were at a greater risk of resistance to at least one drug (OR 1.6). As for new patients, being born in Subsaharan Africa (OR 1.5, 95\% CI 1.04-2.3), but not in Asia, was significantly associated with resistance. In addition, being born in European countries other than France was also associated with resistance to at least one drug (OR 2.3, 95\% CI 1.2-4.6). Factors independently associated with secondary MDR were birth outside of France (OR 5.5) and age <45 yrs (OR 2.0). However, smear status and HIV co-infection were not significantly associated with MDR. Finally, HIV co-infection was the only factor significantly associated with secondary

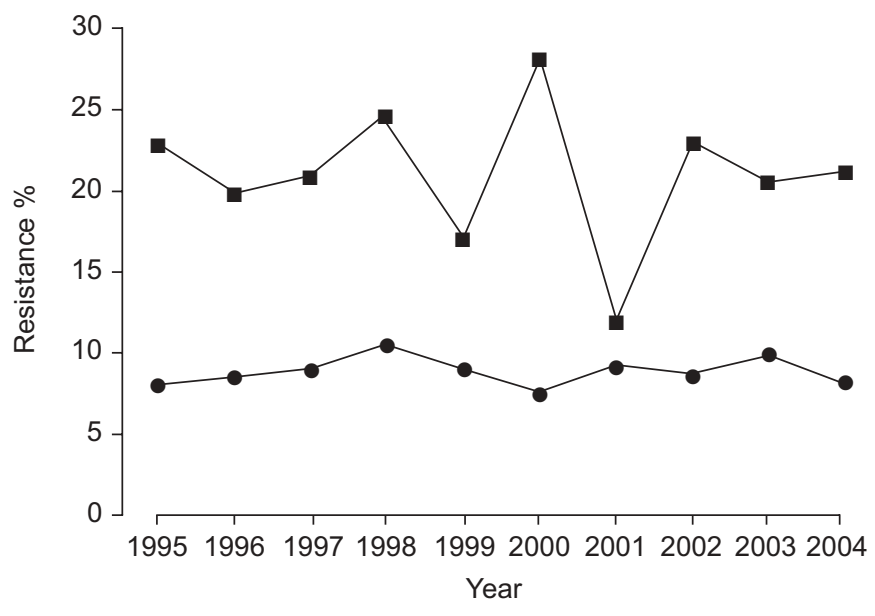

FIGURE 2. Evolution of resistance rates to a minimum of one first-line drug in previously treated
) and previously untreated
RMP monoresistance. All patients harbouring monoresistant strains were HIV-positive.

There was no significant time trend in resistance rates to at least one drug (fig. 2), or to each drug taken separately, among previously treated cases.

\section{DISCUSSION}

Surveillance of TB drug resistance is of major interest for TB control and is used as an indicator for TB treatment quality. In France, the surveillance of drug resistance is conducted by a sentinel network that investigates almost one-third of all culture-positive cases. The network provides two important pieces of information regarding the general epidemiology of TB in France. These are: 1) the increase in the proportion of patients born outside of France; and 2) the increase in the proportion of new cases. The results of the surveillance show that anti-TB drug resistance is not a major health issue in France, at least among never-treated patients. Nevertheless, drug resistance is significantly associated with those born outside of France and HIV co-infection and therefore needs to be carefully monitored in these populations. Finally, a slight upward trend was observed for MDR strains among new patients over the 10-yr period.

In the last 10 yrs, many European countries witnessed changes in the epidemiology of $\mathrm{TB}$, similar to those observed in the current study; a significant decrease in the incidence of TB in the local-born population and an increased proportion of nonlocal-born patients [9]. In France, the national mandatory notification system observed an increase in the incidence of TB in the foreign population since 1999, involving mostly the younger ages (15-39 yrs) [4]. Such a change has to be taken into account for the correct interpretation of resistance data at the national level and particularly to analyse trends. Indeed, it is well established that drug resistance is closely linked to the country of origin of the patient. The current authors observed an increase in the relative proportion of patients born in subSaharan Africa, where the prevalence of resistance has been reported to be higher than in most other parts of the world [10]. Consequently, the observed increase in the prevalence of primary resistance in the current study is likely to be linked to both an increase in the proportion of patients born outside of France and to a change in the country of origin of those patients not born in France.

The proportion of drug resistance among new patients (primary resistance) is an indicator of the transmission of TB strains in a population. In 2004, overall primary resistance rates to INH $(4.2 \%)$ and RMP $(0.9 \%)$ in France were similar to those observed in Belgium (3.7 and $1.0 \%$, respectively), the Netherlands (5.5 and $0.3 \%$, respectively) and Switzerland (5.6 and $0.9 \%$, respectively). However, they were lower than in Germany (7.9 and 1.6\%, respectively), Italy (8.0 and $2.2 \%$, respectively) and the UK (6.9 and $2.2 \%$, respectively) [11]. In the present study, resistance rates among new patients who were born in France were far $<5 \%$, the threshold reported by $\mathrm{WHO}$ as the upper level for good national programmes [12] However, rates exceed $5 \%$ in untreated patients who were not born in France. In addition, two-thirds of the MDR patients were born outside of France, suggesting that exposure occurred outside of France or transmission by other 


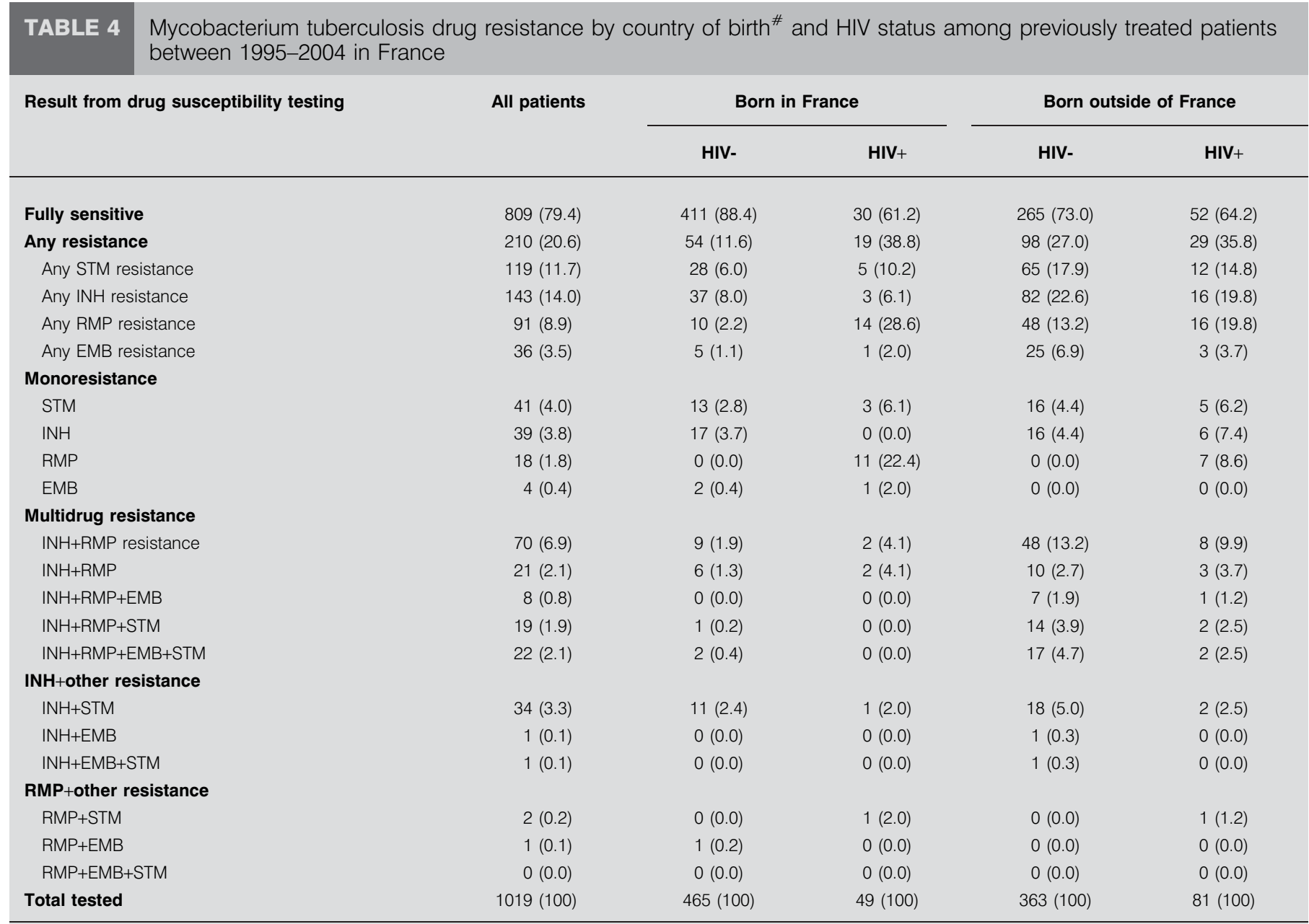

Data are presented as n (\%). STM: streptomycin; INH: isoniazid; RMP: rifampicin; EMB: ethambutol; HIV-: HIV-negative; HIV+: HIV-positive. ${ }^{\#}$ : country of birth was unknown for 61 patients.

non-French-born persons within France. Although patients who were born outside of France may represent a source for resistant strains, it has been shown in two low incidence countries that MDR found in patients born outside of the country they inhabit rarely cause secondary cases in the local population $[13,14]$. The difference in the prevalence of drug resistance in the local and the non-local-born population reinforces the importance of stratifying drug resistance rates by patients' origin as stated in the WHO guidelines [3]. In new patients, a majority of RMP-resistant strains are MDR strains. Therefore, RMP resistance is a good marker for MDR, underlining the usefulness of rapid technologies for assessing resistance to this drug.

As expected, the prevalence of drug resistance in France was far higher among previously treated patients than among untreated patients. Overall secondary resistance rates to INH $(14.0 \%)$ and RMP (8.9\%) in France are close to those observed in Bosnia (14.3 and 7.8\%, respectively), and Portugal (12.8 and $7.4 \%$, respectively) in 2004 . They are lower than the rates observed in Germany (26.1 and 15.2\%, respectively) and Italy (36.4 and 20.5\%, respectively), but higher than those observed in the UK (6.8 and 2.7\%, respectively) or Switzerland (3.4 and
$3.4 \%$, respectively) [11]. The close association between HIV coinfection and RMP or MDR resistance is clear, as previously reported $[6,15,16]$. This may be due to nonadherence to therapy or drug interaction. In contrast to what was observed in the untreated population, monoresistance to RMP is at least as frequent as MDR among HIV co-infected patients. Therefore, RMP resistance cannot be considered as a proxy for MDR among HIV-positive persons who were previously treated for $\mathrm{TB}$, reinforcing the value of a reliable and rapid test for INH resistance, in addition to the currently available rapid test for RMP resistance [17].

Sustaining surveillance over a long time-period, while assuring data quality, is challenging. In the present study, the characteristics of the network have evolved over the $10-y r$ study period. Indeed, the national geographical coverage has been enlarged, increasing the number of patients included in the surveillance. Consequently, the network represents almost one-third of all culture-positive cases reported to the NCR in the last 3 yrs of the surveillance. In the national mandatory notification system, the proportion of patients from the Paris area within France was $48 \%$ in 2000 and $41 \%$ in 2005, and the proportion of pulmonary involvement, French-born patients 
and previously treated patients were $73 \%, 52-55 \%$, and $8-9 \%$, respectively between 2001 and 2005 [18, 19]. These proportions are very close from those observed in the present surveillance network, i.e. $43 \%$ of patients were from the Paris area, $70 \%$ of pulmonary involvement, 53\% of French-born patients (among informed cases) and $8 \%$ of previously treated patients, suggesting an adequate representative of the network. Although no formal evaluation of the network has been performed, it has been shown in a recent study by GUERRIN-TRAN et al. [20] that concordance of data collected through the network and through the mandatory notification system was highly satisfactory.

The changes of the network over time could have induced changes in the population under surveillance and consequently could explain the observed trends. Patients recruited through the laboratories that joined the network over time (new laboratories) had similar characteristics to those included by laboratories participating in the surveillance since the beginning of the programme (original laboratories). In addition, trend analysis performed with data extracted from original laboratories showed similar tendencies to the total population, except for isoniazid resistance. Therefore, trends observed over the 10-yr period are likely to be real and not biased by the addition of new laboratories.

In conclusion, the AZAY-Mycobacteria network provided useful data on the characteristics of tuberculosis in France as well as on resistance to first-line drugs. The sustained efforts of microbiologists over a long time-period allowed the analyses of trends in respect to resistance rates. Drug resistance is not a major issue in patients who were born in France. Observed rates in the current study were close to those estimated in good national programmes. However, close monitoring is needed in the population that were not born in France, especially those born in sub-Saharan Africa, and those previously treated and born in Eastern Europe.

\section{ACKNOWLEDGEMENTS}

Members of the AZAY-Mycobacteria study group involved in tuberculosis diagnosis and data collection are as follows (all France). G. Laurans (CHU d'Amiens, Amiens); M. Chetaou (CHU d'Angers, Angers); G. Couetdic (CHU Jean Minjoz, Besançon); F. Jaureguy (CHU Avicenne, Bobigny); J. TexierMaugein (CHU Haut Lévêque, Pessac, Bordeaux); M-L. Abalain (CHU Morvan, Brest); B. Malbruny (CHU Côte de Nacre, Caen); L. Lebrun (CHU Antoine Béclère, Clamart), L. Deforges, E. Cambau (CHU Henri Mondor, Créteil); J-M. Duez (CHU du Bocage, Dijon); E. Ronco (CHU Raymond Poincaré, Garches); N. Lemaître (CHU de Lille-Hôpital Calmette, Lille); C. Martin (CHU Dupuytren, Limoges); M. Chomarat, M. de Montclos (CHU Lyon-Sud, Lyon); M. Drancourt (CHU Salvator, Marseille); D. Terru (CHU Arnaud de Villeneuve, Montpellier); M. Dailloux (CHU de Nancy, Nancy); P. Bemer (CHU Hôtel Dieu Nantes, Nantes); L. Landraud, D. Sicard (CHU Nice, Nice); C. Pierre, R. Ruimy (CHU Bichat, ParisBichat); S. Coignard (CHU Hôtel-Dieu, Paris); J. Robert, C. Truffot-Pernot, N. Veziris (CHU Pitié-Salpêtrière, Paris); V. Lalande (CHU St-Antoine, Paris); J-L. Hermann (CHU StLouis, Paris); A. Rossier (CHU Tenon, Paris); H. Vu Thien (CHU Trousseau, Paris); G. Agius, A. Bourgoin (CHU de Poitiers, Poitiers); L. Brasme (CHU de Reims, Reims); M. Pestel-Caron (CHU Charles Nicolle, Rouen); A. Carricajo
(CHU St-Etienne, Saint Etienne); R. Bauriaud (CHU Purpan, Toulouse); B. Cattier, P. Lanotte (CHRU de Tours, Tours); M-F. David, D. Mathieu (CHU Paul Brousse, Villejuif).

\section{REFERENCES}

$1 \mathrm{WHO} / \mathrm{IUATLD}$. Antituberculosis drug resistance in the world. Report No 2, prevalence and trends. The WHO/ IUATLD global project on anti-tuberculosis drug resistance surveillance. Geneva, World Health Organization, 2000. Report No.: WHO/CDS/TB/2000.278.

$2 \mathrm{WHO} / \mathrm{IUATLD}$. Guidelines for surveillance of drug resistance in tuberculosis. Int J Tuberc Lung Dis 1998; 2: 72-89.

3 Schwoebel V, ML Moro, CS Lambregts-van Weezenbeek et al. Standardisation of antituberculosis drug resistance surveillance in Europe. Recommendations of a World Health Organization (WHO) and International Union Against Tuberculosis and Lung Disease (IUALD) Working Group. Eur Respir J 2000; 16: 364-371.

4 Che D. Les cas de tuberculose déclarés en France en 2004. [Tuberculosis cases notified in France 2004.] Bull Epidemiol Hebdo 2006; 18: 121-125.

5 Canetti G, Gay P, Le Lirzin M. Trends in the prevalence of primary drug resistance in pulmonary tuberculosis in France from 1962 to 1970: a national survey. Tubercle 1972; 53: 57-83.

6 Robert J, Trystram D, Truffot-Pernot C, Carbonnelle B, Grosset J, AZAY Mycobacteria Study Group. Surveillance of Mycobacterium tuberculosis drug resistance in France, 1995-1997. Int J Tuberc Lung Dis 2000; 4: 665-672.

7 World Health Organization. Guidelines for Surveillance of Drug Resistance in tuberculosis. 2nd Edn. Geneva, WHO, 2003.

8 Heifets L. Susceptibility testing of Mycobacterium tuberculosis to pyrazinamide. J Med Microbiol 2002; 51: 11-12.

9 Borgdorff MW, van der Werf MJ, de Haas PE, Kremer K, van Soolingen D. Tuberculosis elimination in the Netherlands. Emerg Infect Dis 2005; 11: 597-602.

10 Aziz MA, Wright A, Laszlo A, et al. Epidemiology of antituberculosis drug resistance (the Global Project on Anti-tuberculosis Drug Resistance Surveillance): an updated analysis. Lancet 2006; 368: 2142-2154.

11 EuroTB and the national coordinators for tuberculosis surveillance in the WHO European region. Surveillance of tuberculosis in Europe. Report on tuberculosis case notified in 2004. Institut de Veille Sanitaire, Saint Maurice, 2006. www.eurotb.org/rapports/2004.pdf. Date last accessed August 8, 2007.

12 Crofton J, Chaulet $\mathrm{P}$, Maher D. Guidelines for the management of drug resistant tuberculosis 1997. WHO/ TB/96.210. www.who.int/gtb/publications/gmdrt/index. htm Date last accessed: August 8, 2007.

13 Bauer J, Yang Z, Poulsen S, Andersen AB. Results from 5 years of nationwide DNA fingerprinting of Mycobacterium tuberculosis complex isolates in a country with a low incidence of M. tuberculosis infection. J Clin Microbiol 1998; 36: 305-308.

14 Lambregts-van Weezenbeek CSB, Jansen HM, Veen J, Nagelkerke NJD, Sebek MMGG, van Soolingen D. Origin and management of primary and acquired drug-resistant tuberculosis in The Netherlands: the truth behind the rates. Int J Tuberc Lung Dis 1998; 2: 296-302. 
15 Ridzon R, Whitney CG, McKenna MT, et al. Risk factors for rifampin mono-resistant tuberculosis. Am J Respir Crit Care Med 1998; 157: 1881-1884.

16 Munsiff SS, Joseph S, Ebrahimzadeh A, Frieden TR. Rifampin-monoresistant tuberculosis in New York city, 1993-1994. Clin Infect Dis 1997; 25: 1465-1467.

17 Brossier F, Veziris N, Truffot-Pernot C, Jarlier V, Sougakoff W. Performance of the genotype MTBDR line probe assay for detection of resistance to rifampin and isoniazid in strains of Mycobacterium tuberculosis with lowand high-level resistance. J Clin Microbiol 2006; 44: 3659-3664.
18 Cailhol J, Che D, Campese C, Decludt B. Les cas de tuberculose déclarés en France en 2001. [Tuberculosis cases notified in France 2001.] Bull Epidemiol Hebdo 2003; 10-11: 54-57.

19 Antoine D, Che D. Les cas de tuberculose déclarés en France en 2005. [Tuberculosis cases notified in France 2005.] Bull Epidemiol Hebdo 2007; 11: 85-89.

20 Guerrin-Tran E, Thiolet JM, Rousseau C, et al. An evaluation of data quality in a network for surveillance of Mycobacterium tuberculosis resistance to antituberculosis drugs in Ile-de-France region-2001-2002. Eur J Epidemiol 2006; 21: 783-785. 